\title{
Metric analysis of the lumbar region of human vertebral column
}

\author{
M.Ł. Gleinert-Rożek, A. Kosiński, A. Kaczyńska, M. Zajączkowski, W. Kuta, R. Kamiński, G. Piwko \\ Department of Clinical Anatomy, Medical University of Gdansk, Poland
}

[Received: 2 September 2019; Accepted: 21 November 2019]

\begin{abstract}
Detailed measurement of lumbar spine, despite the many years of study, still provides new information, especially due to low back pain, which is increasing and unresolved worldwide health problem. This review includes historical background and evolution of measurement methods. The paper also focused on searching optimal animal model of lumbar spine and summarizes current knowledge and essential tips. In addition, practical application of lumbar metric analysis was presented. This summary is a starting point for further consideration. (Folia Morphol 2020; 79, 4: 655-661)
\end{abstract}

Key words: anatomy, anthropology, morphometry, lumbar spine, low back pain, animal models, comparative anatomy

\section{INTRODUCTION AND APPLICATION OF THE STUDY}

For centuries, the human spine anatomy has been the subject of research in diverse fields of science. In anthropology, this knowledge is the basis for inferring the body structure, style and living conditions of contemporary populations. In clinical practice it means a better understanding of the structure and function of the spine, allowing for more precise clinical diagnosis and surgical treatment of spinal column dysfunctions. Spine-stabilising system design as well as implants improving surgical procedures are both based on measurements of vertebrae structure. In ergonomics and biomechanics, the most up-to-date results are key in the construction process of cutting-edge mathematical digital models of human spine. Aforementioned areas of science exemplify the importance of detailed vertebrae analysis. Interestingly, the lumbar area of the spinal column seems to be particularly important due to the increasing occurrence of disorders in this region.

Low back pain is the leading cause limiting physical activity and notably affecting more and more people around the world at various stages of their lives; therefore, it becomes one of the key modern era health problems, causing an increasing economic burden within the society. Therefore, a detailed lumbar spine examination seems to be particularly important and may contribute to improving many aspects of people's lives $[9,10,18]$.

\section{METHODS OF OBSERVATIONS}

Many studies dealing with lumbar spine are based on the analysis of human cadaver. Such a material enables very detailed measurements of most anatomical vertebrae structures and allows observation of mutual dependencies. However, this type of material is difficult to obtain, especially from the younger population. In addition, any reasoning process related to the lumbar region based on such material is obviously incomplete: one must point out the necessity of using non-invasive systems enabling intravital analysis of this area. Historically, the initial measurements were made using X-ray imaging; however, they were often subject to an error related to the accuracy of calculations based on magnification distance.

Address for correspondence: Dr. A. Kosiński, Department of Clinical Anatomy, Medical University of Gdansk, ul. Dębinki 1, 80-211 Gdańsk, Poland, tel: +48 5834914 20, e-mail: akosinski@gumed.edu.pl 
The introduction of computed tomography (CT) scans made it possible to properly assess the dimensions of vertebral bodies in patients; however, the use of this imaging method is justified only in the case of symptomatic disease while it is not recommended as part of prophylaxis. Studies of healthy population should be based on the analysis of magnetic resonance images (MRI), which is a less invasive study, but also less precise in defining the borders of bone structures $[20,25]$. The most important factor that defines the value of obtained data is the precision of the measurements, which is why the appropriate and possibly cutting-edge methodology should be key when choosing an analytical tool.

\section{THE HISTORICAL BASIS}

Research devoted to the structure of the spine has a long and rich history and a lot of information is to be found in $19^{\text {th }}$-century anatomy textbooks. One of such examples, by Henle [8], published in 1855, already contains detailed information about the vertebral body height anterior ( $\mathrm{VBHa}$ ), which, according to the book, tends to increase from $14 \mathrm{~mm}-\mathrm{C} 3$ to $29 \mathrm{~mm}$ - L5. Thirty years on, Anderson [1] published a study where he found that the VBHa of the L4 equals its vertebral body height posterior (VBHp), while the VBHa of $L 5$ is greater than its VBHp. In addition, the authors documented an increase in the depth of vertebral bodies from L1 to L3 followed by a decrease to the L5 level. However, linear measurements in the context of complex lumbar vertebrae construction, for many authors did not suffice. As a result we may cite Davis' research [5], which confirmed the earlier results of other researchers that the cross-sectional area (CSA) of vertebrae body of L4 was equal to or larger than that on L5. In addition, the following relationship was observed: the relatively small area of L5 body is accompanied by increasing dimensions of the pedicles of spinal arches and transverse processes, which may explain the mechanism of transferring forces from the lumbar region to the pelvic girth. Detailed knowledge of the aforementioned lumbar vertebrae structures was the basis for both theoretical and practical considerations.

A wide-ranging study by Zindrick et al. [26] included analysis of up to 2905 vertebrae, including lumbar vertebrae; although the measurements were restricted to such parameters as height, width, sagittal and transverse angle of the pedicles, as well as the distance to the anterior cortex through pedicle angle axis. It aimed to improve surgical technique by determining the appropriate size of transpedicular screws and insertion axis. Results are important in preventing screw cutout and failure of fixation or neurological injury. In addition, the authors did not observe significant differences between the measurements taken on the basis of CT and X-ray images. The first comprehensive study of the three-dimensional (3D) anatomical structure of lumbar vertebrae was carried out by Panjabi et al. [19] who used X-ray method to document a 3D anatomy of human lumbar vertebrae analysing 60 vertebrae derived from 12 complete spinal columns. The authors noted that the lumbar spine can be naturally subdivided into three areas: thoraco-lumbar transition zone, lumbar-sacral transition zone and the central region. Vertebrae L1 and L2 exhibited features similar to the thoracic segment, which was expressed most notably in size of spinal canal, the width to depth ratios increased from L1 to L3. In addition, the height of the pedicle decreased from $L 1$ to $L 3$, thus continuing the tendency occurring in the lower thoracic segment. Segment $L 3$ was characterised by a relatively narrow spinal canal and the largest area of the vertebral body, as well as the longest spinous process, probably related to the physiological lordosis peak in this segment. Vertebrae L4 and L5 had features similar to those of the sacral spine, mostly expressed as a significant increase in them spinal canal area from L3 to L5 and a sharp increase in the size of the pedicles, due to the increasing load of the lower lumbar region. Such information is fundamental for development of innovative mathematical model of lumbar spine, also allows to better understanding of spine and more exact clinical diagnosis. The methodology used in this study was relatively simple and cost-effective, in contrast to expensive techniques such as $\mathrm{CT}$ and despite low cost allowed to achieve a high degree of measurement accuracy. The small number of samples examined in the study should be considered its main flaw. Also, due to the aforementioned difficulty in obtaining this type of material, only 12 cadaveric specimens were analysed.

The broad study published by Zhou et al. [25] provided extensive data which enabled introduction of an accurate database characterising the lumbar spine of patients reporting the ailments of this area. The analysis involved measurements of 378 lumbar vertebrae, gathered from $126 \mathrm{CT}$ scans. It revealed that the depth and width of the vertebrae body increases 
from $L 3$ to $L 5$; the width of the relevant pedicles alike. In addition, the VBHa remained unchanged within the same region, while VBHp decreased from L3 to L5. The study also determined the average height of the intervertebral discs at each level, with the decreasing tendency in the caudal direction (from L3-L4 to L5-S1). Using the data and its analysis, a lumbar spine model was created, allowing a better understanding of the structure and function of the spine and practical use of these findings in treating patients with lumbar dysfunctions by providing parameters necessary to conservative treatment and also to improve surgical techniques. Measurements performed within the lumbar region tend to provide a good foundation for the development and subsequent production of medical devices, implants as well as in biomechanics and ergonomics. With the development of technology based on such data, digital anatomical models have been created, facilitating spatial reconstruction of the human body. Using the data from up to 590 measurements gathered in previously published studies by various authors, Magee et al. [16] have developed a very accurate, digital model of the axial skeleton. This model was developed for an average age of 27.9 years and an average height of $1758.2 \mathrm{~mm}$; it allowed an average range of mobility to be determined. Due to the growing popularity of such digital human body models in the context of structure and process simulation, it becomes a major challenge to standardise measurements within the skeleton, so that its geometric reconstruction reflects the reality the most.

\section{THE HISTORICAL AND POPULATIONAL CONTEXT}

Structural details of the spine of modern humans allow for comparison of populations in different historical periods. It is a common knowledge that the overall skeletal strength has decreased over the centuries, especially with the recent rapid increase in technological development. Improved health care as well as change in nutrition habits and physical activity, has had a significant impact on the appearance of the modern phenotype, which regardless of reasons differs from the medieval one in terms of biomechanics and function. This particular issue was addressed by Junno et al. [11] the fourth lumbar vertebrae was the subject of analysis based on 91 MRI images of contemporary human vertebrae and 92 skeletons from the Middle Ages (IX-XIII c.). The authors found that over the centuries the height of the vertebral body increased while its width decreased. It seems plausible that these changes may be associated with an overall change in body height. In the old days, the average body height was $171 \mathrm{~cm}$ for men and $158.2 \mathrm{~cm}$ for women whereas today these values equal to $182.7 \mathrm{~cm}$ and $165.5 \mathrm{~cm}$, respectively. The overall vertebral robusticity decreased significantly over time, very likely affecting dimension of vertebral body as well. There is no single explanation for this phenomenon, because the endurance of vertebrae seems to be influenced by both genetic and environmental factors. Probably in the Middle Ages, the quantity and quality of physical loads that the skeleton had to endure was much higher than today. Several studies point out the relationship between individual physical activity level, bone dimension and density. Based on these results, Junno et al. [12] hypothesised that the reduction in vertebral dimensions may be a reflection of changes in the level of physical activity. Therefore the scientists examined the relationship between physical activity in the late adolescence period and strength of the spine expressed in such parameter as vertebral CSA [13]. Surprisingly, in both studies they found that the level of physical activity had no effect on the strength of the lumbar spine, thus the role of other factors such as nutrition or genotype should be taken into account. Knowledge about the factors contributing to vertebral strength could be essential for prevention of vertebral fractures e.g. in osteoporosis.

\section{VERTEBRAL CANAL}

Such analysis would not be complete without the spinal canal evaluation, which is performed with multiple accurate measurements within individual vertebral foramen. These readouts are particularly interesting in the context of spinal canal pathology treatment and can be potentially widely used in the fields of neurology and neurosurgery. For centuries, scientists have been analysing the morphological structure of the spinal canal in the healthy population using a variety of measurement techniques. Unfortunately, also in this case, the comparison of various studies is extremely difficult due to the different methods of measurement as well as anatomical reference points. Notwithstanding, Frostell et al. [6] attempted to estimate the width and depth of the spinal canal within the population, based on previously published works. The dimensions of these parameters are highly dependent on individual features, including body height or torso length. In order to 
standardise the results, the authors created a model where the relative length of the spinal cord and the height of the spine were taken into account and the percentage values of individual spinal segments and vertebrae were determined. For example, the height of the $L 5$ vertebra constitutes $5.5 \%$ of the entire spine. Estimates of such parameters in particular population are extremely important in clinical practice; they are a reference point for the diagnosis and treatment of patients with dysfunctions in this area, for example, suffering from multiple sclerosis have decreased CSA in comparison with healthy controls.

A similar issue was raised by Griffith et al. [7]. The authors developed a reference range for the developmental lumbar spinal canal dimensions - with measurements taken at each lumbar level and at each sex. In addition, it was found that at the L3 level the developmental spinal CSA and depth are smallest, while these parameters increase in the cranial and caudal direction. In taller people, the dimensions of lumbar spinal canal were larger; however, there were no changes observed in its dimensions related to age, weight and body mass index. Such findings are crucial for the studies aiming to determine the developmental spinal stenosis and assess the narrowing of this structure.

Tekin Orha et al. [23] found that degeneration of intervertebral discs and reduction of vertebral body height progressed with age. The comparative observations of spinal canal allowed the conclusion that the depth of the spinal canal may be an important diagnostic parameter of the spinal canal stenosis and an increased antero-posterior dimension of the intervertebral disc seems to be the reason for aggravated pain. Knowledge about the factors related to disc hernitation may be essential to assess the stage of disease progression and choose appropriate treatment method.

The sex-related dimorphism of the spine seems to be an unresolved issue. When comparing the average values for male and female vertebrae, Piontek and Budzyńska [21] concluded that particular differences occur in width and depth of vertebral bodies and the lengths of spinous process and the width of transverse processes. Smaller differences were observed between male and female vertebrae height. In other study sexual dimorphism was evident in the spinal canal CSA - larger in men, although in relation to the vertebral body CSA it was found to be greater in women $[6,7]$.
It should be assumed that the sexual dimorphism of lumbar spine results from the more massive structure of male vertebrae as compared with female counterpart, where it may be associated with the menopause, post-menopausal period as well as age. Nevertheless, these determinants of vertebral sexual dimorphism cannot constitute unambiguous diagnostic elements in determining the sex of the examined subject. Therefore, further research would be most welcome in order to determine obvious differences in age and sex-related spine morphology [21, 23].

Observations of age-related bone changes in bone structure and reduction of bone mineral density, especially in women, led researchers to the hypothesis that the higher risk of vertebral body fractures in older women may be the result of the inability to remodel and adapt to the phenomenon of bone mineral density reduction. To address this question Junno et al. [14] used 181 skeletons from the beginning of the $20^{\text {th }}$ century and $497 \mathrm{MRI}$ images of the current population. The authors took measurements of $L 4$ vertebrae due to its specificity to withstand greater load. They found a moderate relationship between age and vertebral body area growth which was similar in both sexes, therefore no clear, sex-specific mechanism to compensate for the loss of bone mineral density was established. On this basis, the observed more frequent spine fractures in older women cannot be fully explained.

\section{COMPARATIVE ANATOMY}

Implementation of cutting-edge surgical procedures and the development of vertebral implants are preceded by extensive preclinical phase research on human cadavers. Availability of such material is very limited, especially the cadavers of young people, therefore an alternative animal model is still being sought after, especially as an equivalent of desired age groups. Various species were taken into account when looking for the optimal animal model of human vertebral column. The easy access and appropriate dimensions made sheep, deer, calves, pigs, goats and dogs the most commonly studies species. Various differences are evident when comparing the human spine and those of individual animal species. In the lumbar region, the width and depth of human vertebral body as well as the spinal canal are larger than the animal ones. In addition, the mean pedicle angle of the human is lower than in any of the studied animal species. However, there are also convergent features. Like in humans, the sheep 
lumbar spine vertebrae are characterised by a larger width than the depth, creating a typical oval shape within the transverse dimension [24]. On the other hand, the dimensions of lumbar pedicles exhibit the pronounced similarity between the deer and man. In addition, bone mineral density is comparable in both species and this fact may be used in biomechanics, for example when studying the pull out strengths of spinal implants [15]. However, in McLain et al. [17] observed that out of 5 animal species, it is the spine of the miniature pig that most closely reflects the structure of the human spine in terms of its total vertebral height, vertebral body width and spinal canal dimensions. Immature pigs are the best model of such human parameters as the length of transverse processes, depth of vertebral body, pedicle diameters, shape and angle, and also interfacet distance and facet outline. Another alternative to the human model is the calf spine, exhibiting similar characteristics to that of immature pigs. However, both calves and pigs grow very quickly during the first 18 months of life. Individuals included in the experiment at 7-10 months of age, often triple their body weight during the following year, which should be taken into account when planning in vivo studies [3, 17, 22].

Worth mentioning is the study by Busscher et al. [2], where 6 human and 6 porcine spinal columns were subjected to CT and then 16 anatomical measurements were made on each vertebra. Similarities were observed in the vertebral body height, the shape of their end-plates, the shape of the spinal canal and the size of pedicles. In addition, all individual features and dimensions were comparable except the spinal canal depth and the angle of spinous processes. Moreover the authors described similarities in the spinal canal size, the transverse processes length and size of the pedicles. The greatest differences were observed in the dimensions of the intervertebral discs, therefore the porcine spine should not be considered a representative model for testing implants and surgical techniques of this structure. Given the difference in scaling, it is plausible that the porcine spine may be the anatomical equivalent of the human spine in targeted research $[2,4]$. The ideal animal model of the human spine does not exist. Understanding their differences and similarities is the foundation to interpret the research results using such models and may be helpful when selecting an appropriate animal model for in vivo as well as in vitro studies of the spine.

\section{MEASUREMENT STANDARDISATION}

As mentioned before, an important aspect of lumbar spine research is the measurement quality of the structures of this area. Analysis of existing literature indicates significant differences especially between measurement protocols, imaging and image enlargement. The frequent limitations which might have led to flawed results include the study group size as well as the number of samples tested. Magee et al. [16] described this problem in detail in the work devoted to the development of a standardised digital 3D model of the human spine. In addition, further literature analysis reveals a number of inconsistencies and the lack of standardisation of measurements and data recording; moreover, using the same terms to describe different parameters or different terms referring to the same anatomical structure introduces chaos in the interpretation of results. Another issue relates to the geometric reference points used during measurements - very few researchers provide them, which makes it difficult to digitally reconstruct their way of thinking. Standards of human body measurement methods have been developed by the British Standards Institute; among others for the clothing industry, automotive design and computer models used in ergonomics. Intriguingly, such standards have not been established for measurement methods related to human anatomy.

The recommendations presented by Magee et al. [16] were based on the integration of industrial design standards and engineering methodology. Anatomical terminology requires standardisation and consistency in the context of specific measurements of different areas. Unification of nomenclature would prevent problems with data interpretation (Table 1). Determining the recommended scaling parameter, e.g. growth, would allow comparative analysis.

In addition, the measurement report should contain information on the reference points and spatial position of the examined objects. It is suggested that the centre line of the body is the main reference point in the frontal plane. Anatomy is not characterised by the absolute symmetry, therefore the measurements of the lateral points in relation to the median line should be quantified in both positive and negative values, according to current standards (spinal flexion $[+]$, extension $[-]$, lateral bending to the right $[+]$ and left [-]). Again the Magee group [16] indicated literature examples of the good practice. Moreover, 
Table 1. Vertebrae measurement descriptions

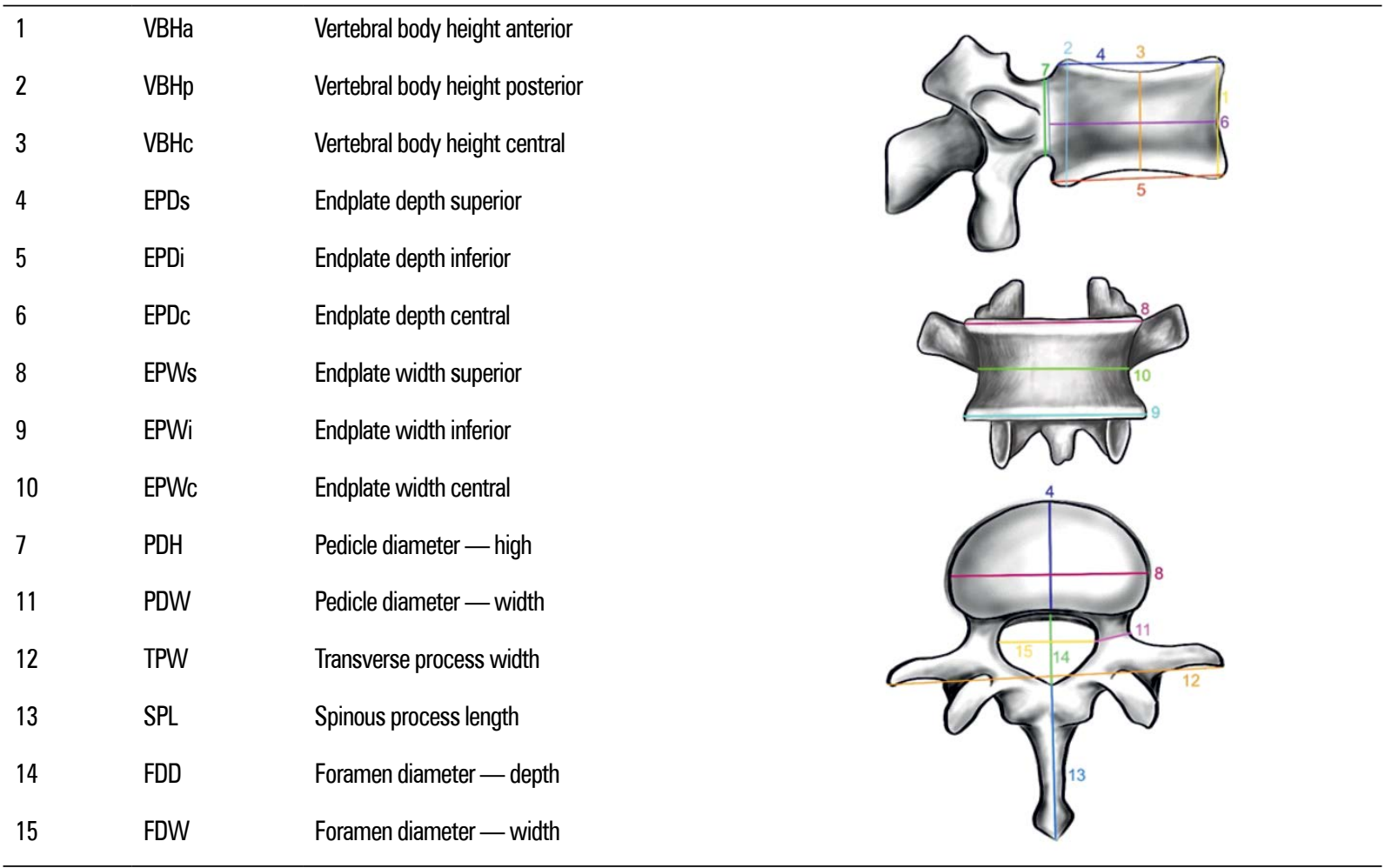

these authors suggested additional guidelines to improve the communication protocol and thus help create further 3D digital reconstructions of human skeleton.

\section{CONCLUSIONS}

Anatomical studies of the lumbar spine, although practiced for many years, still provide new information and inspire further observation. Getting to know the structure and function is becoming more and more important in everyday clinical practice. This is particularly important in the context of lumbar pain which is a growing health problem and the main reason for limiting physical activity and seeking specialist medical care. Due to the complex structure, heterogeneous aetiology and multitude of therapeutic techniques, treating dysfunctions of this area is extremely difficult. Optimal solutions, where the patients would receive a satisfying long-term therapeutic effect, are still being sought after. The issues of the lumbar spine presented in this paper, an outline of the results so far, as well as essential tips, may constitute the starting point for further research.

\section{REFERENCES}

1. Anderson RJ. Observations on the Diameters of Human Vertebrae in Different Regions. J Anat Physiol. 1883; 17(Pt 3): 341-344, indexed in Pubmed: 17231480.

2. Busscher I, Ploegmakers JJW, Verkerke GJ, et al. Comparative anatomical dimensions of the complete human and porcine spine. Eur Spine J. 2010; 19(7): 1104-1114, doi: 10.1007/s00586-010-1326-9, indexed in Pubmed: 20186441.

3. Cotterill PC, Kostuik JP, D'Angelo G, et al. An anatomical comparison of the human and bovine thoracolumbar spine. J Orthop Res. 1986; 4(3): 298-303, doi: 10.1002/ jor.1100040306, indexed in Pubmed: 3734937.

4. Dath R, Ebinesan AD, Porter KM, et al. Anatomical measurements of porcine lumbar vertebrae. Clin Biomech (Bristol, Avon). 2007; 22(5): 607-613, doi: 10.1016/j. clinbiomech.2007.01.014, indexed in Pubmed: 17360085.

5. Davis PR. Human lower lumbar vertebrae: some mechanical and osteological consideration. J Anat. 1961; 95 : 337-344.

6. Frostell A, Hakim R, Thelin EP, et al. A Review of the Segmental Diameter of the Healthy Human Spinal Cord. Front Neurol. 2016; 7: 238, doi: 10.3389/fneur.2016.00238, indexed in Pubmed: 28066322.

7. Griffith JF, Huang J, Law SW, et al. Population reference range for developmental lumbar spinal canal size. Quant Imaging Med Surg. 2016; 6(6): 671-679, doi: 10.21037/ qims.2016.12.17, indexed in Pubmed: 28090445. 
8. Henle J. Handbuch der systematischen Anatomie des Menschen. Braunschweig 1855.

9. Hoy D, Brooks $P$, Blyth $F$, et al. The Epidemiology of low back pain. Best Pract Res Clin Rheumatol. 2010; 24(6): 769-781, doi: 10.1016/j.berh.2010.10.002, indexed in Pubmed: 21665125.

10. Hoy DG, Smith E, Cross M, et al. The global burden of other musculoskeletal disorders: estimates from the Global Burden of Disease 2010 study. Ann Rheum Dis. 2014; 73(8): 1462-1469, doi: 10.1136/annrheumdis-2013-204680, indexed in Pubmed: 24590181.

11. Junno JA, Niskanen M, Nieminen MT, et al. Temporal trends in vertebral size and shape from medieval to modern-day. PLoS One. 2009; 4(3): e4836, doi: 10.1371/ journal.pone.0004836, indexed in Pubmed: 19279681.

12. Junno JA, Paananen M, Karppinen J, et al. Influence of physical activity on vertebral size. Osteoporos Int. 2011; 22(1): 371-372, doi: 10.1007/s00198-010-1252-z, indexed in Pubmed: 20419291.

13. Junno JA, Paananen M, Karppinen J, et al. Influence of physical activity on vertebral strength during late adolescence. Spine J. 2013; 13(2): 184-189, doi: 10.1016/j. spinee.2012.11.049, indexed in Pubmed: 23332389.

14. Junno JA, Paananen M, Karppinen J, et al. Age-related trends in vertebral dimensions. J Anat. 2015; 226(5): 434-439, doi: 10.1111/joa.12295, indexed in Pubmed: 25913516.

15. Kumar N, Kukreti S, Ishaque M, et al. Anatomy of deer spine and its comparison to the human spine. Anat Rec. 2000; 260(2): 189-203, doi: 10.1002/1097-0185 (20001001)260:2<189::AID-AR80>3.0.CO;2-N, indexed in Pubmed: 10993955.

16. Magee J, McClelland B, Winder J. Current issues with standards in the measurement and documentation of human skeletal anatomy. J Anat. 2012; 221(3): 240-251, doi: 10.1111/j.1469-7580.2012.01535.x, indexed in Pubmed: 22747678.

17. McLain RF, Yerby SA, Moseley TA. Comparative morphometry of L4 vertebrae: comparison of large animal models for the human lumbar spine. Spine (Phila Pa 1976). 2002;
27(8): E200-E206, doi: 10.1097/00007632-20020415000005, indexed in Pubmed: 11935119.

18. Meucci RD, Fassa AG, Faria NM. Prevalence of chronic low back pain: systematic review. Rev Saude Publica. 2015; 49: 1-10, doi: 10.1590/S0034-8910.2015049005874, indexed in Pubmed: 26487293.

19. Panjabi MM, Goel V, Oxland T, et al. Human lumbar vertebrae. Quantitative three-dimensional anatomy. Spine (Phila Pa 1976). 1992; 17(3): 299-306, doi: 10.1097/00007632199203000-00010, indexed in Pubmed: 1566168.

20. Pierro A, Cilla S, Maselli G, et al. Sagittal normal limits of lumbosacral spine in a large adult population: a quantitative magnetic resonance imaging analysis. J Clin Imaging Sci. 2017; 7: 35, doi: 10.4103/jcis.JCIS_24_17, indexed in Pubmed: 28904831.

21. Piontek J, Budzyńska J. Zmiennoość cech metrycznych kręgosłupa. Przegl Antropol. 1972; 38: 17-26.

22. Sheng $S R$, Wang $X Y, X u H Z$, et al. Anatomy of large animal spines and its comparison to the human spine: a systematic review. Eur Spine J. 2010; 19(1): 46-56, doi: 10.1007/ s00586-009-1192-5, indexed in Pubmed: 19876658.

23. Tekin Orha A, Dalcik C, Ilbay K. Evaluation of anthropometric characteristics due to sex and age variables of lumbar vertebra and spinal canal. Anthropologist. 2016; 24(2): 617-622, doi: 10.1080/09720073.2016.11892056.

24. Wilke HJ, Kettler A, Wenger $\mathrm{KH}$, et al. Anatomy of the sheep spine and its comparison to the human spine. Anat Rec. 1997; 247(4): 542-555, doi: 10.1002/(SICI)10970185(199704)247:4<542::AID-AR13>3.0.CO;2-P, indexed in Pubmed: 9096794.

25. Zhou SH, McCarthy ID, McGregor AH, et al. Geometrical dimensions of the lower lumbar vertebrae: analysis of data from digitised CT images. Eur Spine J. 2000; 9(3): 242-248, doi: 10.1007/s005860000140, indexed in Pubmed: 10905444.

26. Zindrick MR, Wiltse LL, Doornik A, et al. Analysis of the morphometric characteristics of the thoracic and lumbar pedicles. Spine (Phila Pa 1976). 1987; 12(2): 160-166, doi: 10.1097/00007632-198703000-00012, indexed in Pubmed: 3589807. 